\title{
Dynamique des extrêmes hydrologiques du système Chari-Logone et risques naturels dans la région de l'extrême-nord du Cameroun
}

\author{
Fréderic Saha $^{1}$, Mesmin Tchindjang ${ }^{1}$, Jean-Guy Dzana ${ }^{1}$, and Djasrabé Nguemadjita ${ }^{2}$ \\ ${ }^{1}$ Département de Géographie de l'Université de Yaoundé 1, Yaoundé, Cameroun \\ ${ }^{2}$ Direction des ressources en eau et de la météorologie, N'Djamena, Tchad
}

Correspondence: Fréderic Saha (fredericsaha@yahoo.fr)

Published: 16 November 2021

\begin{abstract}
Résumé. Le système Chari-Logone est principal cours d'eau drainant l'Extrême-Nord du Cameroun. À l'image des autres parties du bassin versant de ce cours d'eau, cette région a connu plusieurs inondations catastrophiques. Cet article examine la dynamique des extrêmes hydrologiques du Logone et du Chari à travers les stations de Bongor et N'Djamena en prenant en compte le contexte des changements climatiques et l'occurrence des risques hydro-climatiques. Les analyses portent essentiellement sur les données journalières maximum (Qmax) et minimum (Qmin) sur la période 1960-2015. Les logiciels XLStat et ChronoStat ont permis de calculer un ensemble d'indices. Il en ressort que les Qmax présentent une rupture en 1971 aux deux stations marquant la fin de la période humide. Les Qmin de Bongor ont connu deux ruptures successives en 1994 et en 2007. Les Qmin de N'Djamena par contre ont connu une rupture en 1986. Le coefficient de tarissement est globalement faible $(0,020 /$ jour en moyenne). L'occurrence des inondations catastrophiques est très contrastée, car seulement $30 \%$ s'inscrivent dans les années excédentaires. Cela témoigne du rôle important que jouent les autres facteurs de construction du risque comme la pédologie, la pluviométrie et la vulnérabilité socioéconomique.
\end{abstract}

\section{Introduction}

Pendant le XXème siècle et le début du siècle en cours, l'Afrique a connu plusieurs séquences hydro-climatiques (Lévêque, 2006). Les années de la période de faibles écoulements entre 1970-1990 par exemple ont connu les étiages les plus sévères (Olivry, 1997). De nombreux pays (Mali, Niger, Tchad, etc.) avaient alors eu des difficultés supplémentaires dans l'approvisionnement en eaux pour différents besoins (boisson humaine et animale, irrigation, etc.). Pour les périodes d'excédents, les crues sont parfois à l'origine d'inondations catastrophiques. Il faut remarquer que ces périodes ne sont pas toujours homogènes. En période de déficits, se mêlent parfois les années de forts écoulements et inversement. En République Centrafricaine, Nguimalet (2017) met en exergue une importante dynamique des débits extrêmes de l'Oubangui entre 1911-2014. L'évolution de ce cours d'eau suit globalement les tendances hydro-climatiques qu'a connues l'Afrique centrale et de l'Ouest. Les débits extrêmes
Qmin et Qmax ont eu de graves conséquences dans la ville de Bangui. En zone tropicale sahélienne, les extrêmes de nombreux cours d'eau furent encore plus marqués avec occurrence de sècheresses et inondations catastrophiques dans de nombreux pays (Olivry, 1997).

Le bassin endoréique du Lac Tchad a durement subi la sècheresse qui a débuté en 1970. On parle d'une diminution de près de $90 \%$ de la superficie du lac. Cela étant dû au déficit d'alimentation; notamment le Logone-Chari qui est la principale source d'alimentation de ce lac (Olivry et al., 1996). Dans le même bassin, quelques crues exceptionnelles ont été à l'origine de graves inondations au Tchad et au Cameroun (OCHA, 2012). Cet article s'intéresse au sous-bassin du Logone et au bassin du Chari. L'objectif est d'évaluer la dynamique de ce système hydrographique sous l'angle des débits extrêmes (Qmin et Qmax). Les résultats de cette dynamique sont mis en corrélation avec l'occurrence et l'ampleur des dégâts des catastrophes naturelles (inondations et 
sècheresses) que la région de l'Extrême-Nord du Cameroun a connues pendant la période d'observation (1977-2017).

\section{Données et méthodes}

Près de $90 \%$ de la région de l'Extrême Nord du Cameroun fait partie du grand bassin hydrologique du lac Tchad en zone tropicale (Olivry, 1986) (Fig. 1). On peut y distinguer trois nuances climatiques : le climat tropical d'altitude qui règne essentiellement sur l'Adamaoua au Cameroun où le Logone prend sa source. Les précipitations moyennes se situent entre $1300-1500 \mathrm{~mm}$. La seconde nuance climatique est soudanienne avec $1000 \mathrm{~mm}$ de pluies en moyenne par an. La partie au nord est le domaine du climat soudano sahélien avec des précipitations qui diminuent selon un gradient sudnord.

Les données de débits de base utilisées concernent la période 1955-2016 (61 ans). Pour chacune des stations, quelques années sont lacunaires. Ces données proviennent d'une part de l'ouvrage "fleuves et rivières du Cameroun » de Olivry (1986), d'autre part de la Commission du Bassin du Lac Tchad (CBLT, 2010) et enfin de la Direction des Ressources en Eau et de la Météorologie (DREM) au Tchad.

Les valeurs annuelles ont été calculées du 1er avril au 31 mars, ce qui correspond à l'année hydrologique. Les traitements et analyses concernent spécifiquement les modules annuels et les débits journaliers extrêmes (maximum et minimum). La variabilité interannuelle du régime hydrologique est appréciée à travers les variations des différents indices. Le coefficient de tarissement (Lang et Gille, 2006) permet de juger de l'intensité des sècheresses hydrologiques, lorsque le débit n'est plus soutenu que par la vidange de la nappe phréatique. La puissance des écoulements de crue est traduite par le coefficient A de Pardé (Loup, 1965). L'indice d'irrégularité ( $Q_{\max }$ annuel $/ Q_{\min }$ annuel) mesure l'écart entre les débits les minima et les maxima annuels (Nguimalet, 2017).

Dans l'optique de détecter les tendances dans la distribution des données annuelles, nous avons appliqué différents tests d'homogénéité sur KhronoStat 1.01 et XLStat. Il s'agit des tests de Pettitt, d'Hubert et de la méthode Bayésienne de Lee-Heghinian. Les données sur les risques naturels (sècheresses et inondations) dans la région de l'Extrême-Nord du Cameroun sont issues des archives du service du gouverneur. Les variables retenues sont les dates d'occurrence des catastrophes et les dégâts enregistrés (pertes en vies humaines, personnes déplacées, pertes matérielles).

\section{Résultats et analyses}

\subsection{Dynamique des extrêmes hydrologique du Logone et du Chari à travers quelques indices}

\subsubsection{Indice d'irrégularité}

La moyenne interannuelle de l'indice d'irrégularité est de $R=44,5$ à Bongor (1955-2016). Il présente une légère tendance générale à la baisse. Pour une moyenne de 48,6 entre 1955-1988, on est passé à 38,5 pour la période 1989-2016 soit une baisse de $20 \%$. Globalement, cela traduit une réduction de l'écart entre $Q_{\min }$ et $Q_{\max }$.

Pour ce qui est de N'Djamena, le coefficient $R$ moyen est de 67,4 (1955-2016). Le maximum de la série est de 668 pour l'année 1988-1989 et le minimum est de 13 correspondants à l'année 1963-1964. Avant la rupture de 1971-1972 le coefficient $R$ était de 29 ; il est passé à 86,5 pour la période 1972-2016; soit une augmentation de $246 \%$.

\subsubsection{Coefficient de tarissement}

Le temps moyen de tarissement est de 190 jours sur le Logone à Bongor. Cela se passe entre le 23 septembre et le 20 mars. Cette moyenne cache une forte variabilité interannuelle. Le coefficient moyen de tarissement interannuel se situe alors à $0,019 /$ jour. Le coefficient le plus élevé est 0,031 /jour correspondant à l'année 1955-1956. Le plus faible est celui de l'année 1982-1983 (0,012/jour). Le coefficient d'asymétrie est de 0,56 ce qui traduit un étalement de la série de données à droite de la moyenne. Les données de N'Djamena sur le Chari présentent un coefficient de tarissement moyen plus élevé qu'a Bongor ( $0,018 /$ jour $)$.

\subsection{Risques hydro-climatiques dans la région de l'Extrême-Nord du Cameroun}

\subsubsection{Différents aspects de la sècheresse hydrologique dans l'Extrême-Nord du Cameroun}

Les données de débits journaliers du système LogoneChari (Bongor et N'Djamena) mettent en évidence quelques aspects de la sècheresse hydrologique affectant la région de l'Extrême-Nord du Cameroun :

- les sècheresses s’étendant sur plusieurs années consécutives;

- les déficits de l'écoulement total annuel;

- les déficits d'étiage et

- le niveau des hautes eaux par rapport à la moyenne.

Pour la station de N'Djamena, le module (1955-2016) est de $903 \mathrm{~m}^{3} \mathrm{~s}^{-1}$. La période 1955-1971 $\left(1304 \mathrm{~m}^{3} \mathrm{~s}^{-1}\right.$ en moyenne) est de $44 \%$ plus élevée que cette moyenne générale. À cette période succède la sècheresse qui se poursuit 


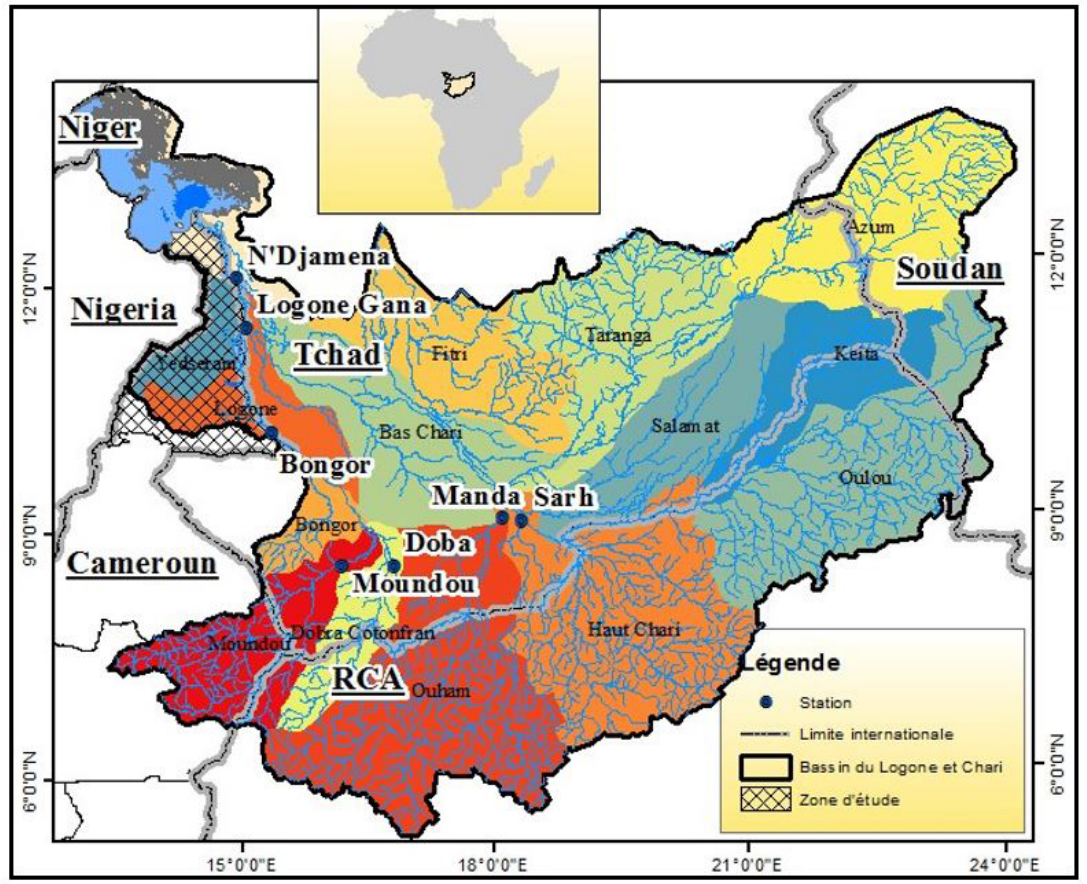

Figure 1. La région de l'Extrême-Nord du Cameroun dans le bassin du Logone-Chari (C CBLT, 2010).

jusqu'à la fin de la série avec des niveaux de sévérité différents. De 1972-1998, le déficit d'écoulement est le plus élevé : $27 \%$. La station de Bongor présente un module de $457 \mathrm{~m}^{3} \mathrm{~s}^{-1}$ (1955-2016). Une sècheresse persistante commence en 1977-1978. Elle se maintient jusqu'en 1994-1995 lorsque la sévérité baisse.

Pour les données de N'Djamena, les excédents de $Q_{\max }$ pendant les années déficitaires étaient récurrentes (19751976, 1976-1977, 1989-1990, etc.). La Fig. 2 présente un indice synthétique de sècheresse résultant de l'addition des variables centrées réduites des modules, $Q_{\min }$ et leurs $Q_{\max }$. Les années de sècheresse se caractérisent par un résultat négatif.

Le contexte socio-économique de l'Extrême-Nord en fait une région très vulnérable aux risques naturels en général et à la sècheresse en particulier. En effet, l'insécurité alimentaire affecte $18 \%$ de la population (DSCN, 2002). Il s'agit aussi de la région la plus pauvre du Cameroun avec 74,3\% de la population vivant en dessous du seuil de pauvreté (INS, 2015). Près de $60 \%$ de la population dépend des sources non aménagées pour l'eau de boisson. L'agriculture (pluviale, irriguée et de contre saison) de subsistance représente $65 \%$ de l'activité économique de la région (DSCN, 2002). Il en est de même de l'activité pastorale non mécanisée. C'est dans ces conditions que les déficits hydrologiques des décennies 1970 et 1980 ont occasionné de véritables catastrophes dans la région de l'extrême nord. Pour les deux décennies les populations l'aggravation : des pénuries en denrées alimentaires, des épidémies de méningite, des pénuries
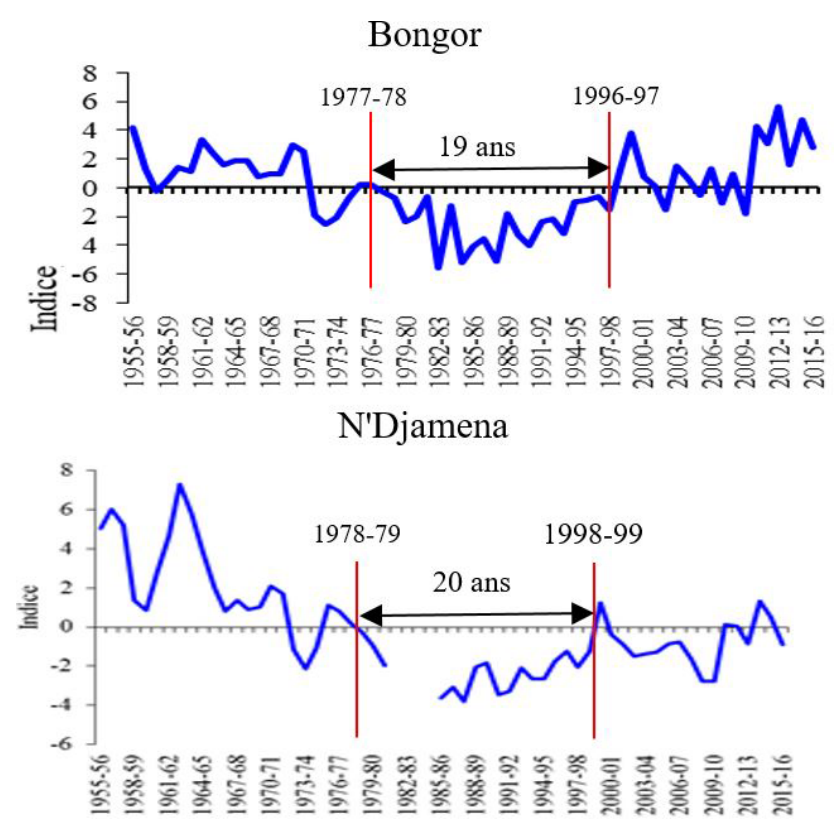

Figure 2. Valeurs de l'indice synthétique de sècheresse hydrologique du Logone à Bongor et du Chari à N'Djamena.

d'eau potable, des invasions de criquets, du tarissement des mares d'eau, de la dégradation des pâturages, de la désertification des terres. Par l'indice de rendement agricole espéré (IRESP), L'Hôte (2000) situe à 27,2\% en moyenne la baisse de rendement sur les 10 cultivars les plus semés dans 
Tableau 1. Facteurs hydrologiques et inondations majeures dans la région de l'Extrême-Nord du Cameroun.

\begin{tabular}{|c|c|c|c|c|c|c|c|c|c|c|}
\hline \multirow[t]{3}{*}{ Année } & \multicolumn{8}{|c|}{ Variable centrée réduite des indices } & \multirow[t]{3}{*}{ Arrondissement les plus affectés } & \multirow[t]{3}{*}{ Principaux dégâts } \\
\hline & \multicolumn{2}{|c|}{$Q_{\text {moyen }}$} & \multicolumn{2}{|c|}{$Q_{\max }$} & \multicolumn{2}{|c|}{ DCC } & \multicolumn{2}{|c|}{ Coefficient $A$} & & \\
\hline & B & $\mathrm{N}$ & B & $\mathrm{N}$ & B & $\mathrm{N}$ & $\mathrm{B}$ & $\mathrm{N}$ & & \\
\hline 2015-2016 & 0,16 & 0,1 & 1,7 & $-1,1$ & 0,9 & $-1,1$ & 1,6 & $-1,1$ & Maroua, Gazawa et Ndoukoula & 5 morts, 6200 sans abris \\
\hline 2014-2015 & 2,37 & 0,01 & & 0,3 & 0,7 & 0,4 & & 0,4 & Zina et Kaikai & 168 sans abris \\
\hline 2012-2013 & 2,76 & $-0,9$ & 2,3 & 0,8 & 2,1 & 0,9 & 2,3 & 1 & Mayo Danay et Logone et Chari & 20 morts; 20000 sans abris \\
\hline 2010-2011 & 0,49 & $-1,07$ & & 0,5 & & 0,59 & & 0,4 & Pouss & 12 morts; 3000 sans abris \\
\hline 2007-2008 & $-0,86$ & $-0,64$ & $-0,6$ & $-0,2$ & 0,3 & $-0,1$ & $-0,6$ & $-0,2$ & Mayo Danay & 8 morts; 4421 sans abris \\
\hline 2006-2007 & 0,37 & 0,43 & 0,3 & -1 & $-0,9$ & $-1,0$ & $-0,3$ & -1 & Maroua & 2 morts; 1042 sans abris \\
\hline 2005-2006 & $-0,41$ & $-0,88$ & $-0,1$ & $-0,1$ & $-0,2$ & $-0,1$ & $-0,1$ & $-0,1$ & Maroua & 14 morts; 453 sans abris \\
\hline 2000-2001 & $-0,16$ & 0,2 & $-0,6$ & $-0,7$ & 1,6 & $-0,7$ & $-0,6$ & $-0,8$ & Maroua & 17 morts; 206 sans abris \\
\hline 1997-1998 & $-0,54$ & $-0,26$ & $-0,6$ & $-1,3$ & $-0,7$ & $-1,2$ & $-0,6$ & $-1,3$ & Maroua & 7 morts; 248 sans abris \\
\hline 1994-1995 & $-0,49$ & $-1,12$ & 0,1 & $-0,4$ & 0,2 & $-0,3$ & 0,1 & $-0,4$ & Maroua, Mokolo, Kousseri & 41 morts; 1004 sans abris \\
\hline 1991-1992 & $-0,98$ & $-1,4$ & $-0,5$ & $-0,8$ & $-0,6$ & $-0,7$ & $-0,5$ & $-0,8$ & Maga et Kousseri & 2 morts; 200 sans abris \\
\hline 1988-1989 & $-0,28$ & $-1,6$ & $-0,2$ & 0,7 & $-0,2$ & 0,9 & $-0,2$ & $-0,8$ & Maroua et Kousseri & 10 morts; 165 sans abris \\
\hline 1977-1978 & $-0,33$ & 0,07 & 0,4 & 0,1 & $-0,1$ & 0,2 & 0,5 & 0,1 & Maroua & 7 morts \\
\hline
\end{tabular}

$\mathrm{B}=$ Bongor. $\mathrm{N}=\mathrm{N}$ 'Djamena.

la zone de Kousseri-N'Djamena en comparant les périodes 1950-1967 et 1968-1985. Ce pourcentage est de 2,2\% dans la zone de Maroua. Il faut remarquer que dans son approche, L'Hôte (2000) se fonde essentiellement sur les données pluviométriques et donc sur la diminution des précipitations, laquelle a affecté plus sévèrement la zone la plus septentrionale. En réponse, le gouvernement du Cameroun avait mis en place un ensemble de mesures pour assister les populations.

\subsubsection{Paramètres hydrologiques et inondation dans l'Extrême-Nord du Cameroun}

Les inondations dans la région de l'Extrême-Nord du Cameroun relève d'un ensemble de facteurs physiques et humains (relief plat, pluviométrie concentrée sur trois mois, sols argileux peu perméables, mauvaise qualité du bâti, précarité générale des modes de vie, etc.). Ainsi, l'eau qui inonde provient directement des précipitations ou alors du débordement des cours d'eau. En abordant l'aspect hydrologique de ce phénomène, les excédents sur les modules et de débits de pointes donnent des perspectives d'analyse. En plus de ces deux variables, il est tout aussi adéquat de s'intéresser aux débits égalés ou dépassés pendant 10 jours par an ou débits caractéristiques de crues (DCC). La moyenne des DCC est de $1714 \mathrm{~m}^{3} \mathrm{~s}^{-1}$ sur le Logone (Bongor) et $2604 \mathrm{~m}^{3} \mathrm{~s}^{-1}$ sur le Chari (N'Djamena). À l'image des autres paramètres de ces cours d'eau, les DCC sont très variables à l'échelle interannuelle. Les valeurs les plus élevées se situent au début de la série d'observation (avant 1971). Toutefois, à Bongor, c'est l'année 2012-2013 qui présente le maximum $\left(2710 \mathrm{~m}^{3} \mathrm{~s}^{-1}\right)$; soit un excédent de $58 \%$. Pour les deux stations, on remarque un retour d'années humides avec un DCC excédentaire à partir des années 2000.

Les années d'inondations catastrophiques ne se caractérisent pas toujours par des débits moyens au-dessus du module, et/ou des DCC et débits de pointe supérieurs aux moyennes interannuelles. La distribution de la puissance de crue (coefficient de Pardé) ne permet pas non plus de tirer des conclusions claires. Le Tableau 1 présente quelques exemples d'inondations catastrophiques dans la région de l'Extrême-Nord du Cameroun en relation avec les variables hydrologiques du Logone et du Chari pour les stations de Bongor et de N'Djamena.

Il se dégage du Tableau 1 que seulement $23 \%$ (3/13) des inondations dans la région de l'Extrême-Nord du Cameroun surviennent pendant les années humides lorsqu'on considère les Q moyens, les débits de pointe et les DCC du Logone et du Chari. Ces années sont les plus récentes (2010-2011, 2012-2013 et 2014-2015).

\section{Discussion}

Nguimalet (2017) analysant les données de 1911-2014 sur l'Oubangui, met en exergue une tendance à la baisse des $Q_{\min }$ et $Q_{\max }$. La rupture de 1971 ressort aussi des analyses de cet auteur. La même tendance a été observée sur le Sénégal et l'ensemble des bassins Ouest africains (Olivry, 1997). Il faut remarquer que sur les grands fleuves africains en général, le comportement des $Q_{\max }$ est identique aux tendances observées sur les débits moyens annuels (Olivry et al., 1998). La variabilité des $Q_{\min }$ se conforme très peu à cette tendance générale. Les cours d'eau d'Afrique de l'Ouest ont subi plus sévèrement la péjoration des écoulements par rapport à l'Afrique centrale (Olivry et al., 1998). En effet, sur le Sénégal, le déficit est de 57\% entre 1971-1993 avec un regain d'humidité à partir de 1994 (Cisse et al., 2014).

Olivry et al. (1997) relève une faible puissance des crues sur les fleuves d'Afrique tropicale (coefficients $A$ de PARDÉ de 6 à 28 pour le Niger, de 2 à 20 pour le Sénégal). On peut évoquer l'immensité des bassins de ces cours d'eau comme 
facteur explicatif. L'année 1984, qui a connu la plus faible puissance de crue sur le Logone et le Chari, est identifiée comme la plus déficitaire de la période sèche sur plusieurs cours d'eau d'Afrique (Olivry et al., 1996). Les tarissements du Logone et du Chari sont nettement plus lents (190 jours) que ceux des fleuves d'Afrique de l'Ouest où deux mois seulement séparent $Q_{\max }$ de $Q_{\min }$ (Olivry et al., 1994). La situation du Logone et du Chari s'apparente plus à la tendance des cours d'eau d'Afrique équatoriale, comme l'Oubangui (affluent du Congo) où Nguimalet (2017) trouve pratiquement la même valeur moyenne du coefficient de tarissement $(0,020 /$ jour $)$.

\section{Conclusion}

Il ressort de cet article que du point de vue temporel, la tendance majeure est celle de la baisse des débits de pointe à partir de 1971-1972 évaluée à 25,5\% à Bongor et $36 \%$ à N'Djamena. La tendance des minimums d'écoulement est plus contrastée. La baisse des débits de pointe se répercute sur la puissance des crues. La régularité ou l'irrégularité du rapport entre $Q_{\max }$ et $Q_{\min }$ se situe entre 13 et 668 ce qui traduit une forte variabilité.

En considérant les variables centrées réduites, de 19771978 à 1996-1997 pour Bongor et de 1978-1979 à 19981999 pour N'Djamena, tous les indices $\left(Q_{\min }, Q_{\max }\right.$, coefficient de tarissement et puissance de crues) étaient négatifs. Cette période correspond à la réalité locale de la sècheresse hydrologique ayant affecté l'Afrique. Il faut y ajouter d'autres années et des périodes plus courtes qui ont aussi connu des déficits d'écoulement. Du point de vue des crues, il ressort des analyses que ce ne sont pas toujours pendant les années excédentaires que les inondations catastrophiques sont enregistrées.

Disponibilité des données. Les données utilisées sont accessibles uniquement auprès des auteurs.

Collaborateurs. Cet article est redigé par FS sous la supervision de MT et JGD. DN a collecté les données hysrologiques et participé a leurs traitements.

Intérêts concurrents. Les auteurs déclarent qu'ils n'ont aucun conflit d'intérêts.

Clause de non-responsabilité. Publisher's note : Copernicus Publications remains neutral with regard to jurisdictional claims in published maps and institutional affiliations.
Déclaration du numéro spécial. This article is part of the special issue "Hydrology of Large River Basins of Africa". It is a result of the 4th International Conference on the "Hydrology of the Great Rivers of Africa", Cotonou, Benin, 13-20 November 2021.

Remerciements. Nous exprimons notre gratitude a l'endroit de IAHS SYSTA award qui a financé la présentation et la discussion des resultats de cette étude à la 4ème Conférence de Friend Water sur l'hydrologie des grands fleuves d'Afrique qui s'est tenue du 13 au 20 novembre 2021 à Cotonou.

\section{Références}

Bureau de la coordination des affaires humanitations des Nations Unies (OCHA) : Note de synthèse : impact des inondations. Afrique de l'Ouest et du Centre, septembre 2012, Rapport du Bureau de la Coordination des Affaires Humanitaires des Nations Unies, 5 pp., en ligne, 2012.

Cisse, M. T., Sambou, S., Dieme, Y., Diatta, C., et Bop, M. : Analyse des écoulements dans le bassin du fleuve Sénégal de 1960 à 2008, Revue des Sciences de l'Eau, vol. 27, no. 2, 167-187, https://doi.org/10.7202/1025566ar, 2014.

Commission du bassin du lac Tchad (CBLT) : Création et vulgarisation d'une charte de l'eau du lac Tchad. Phase 1 - Diagnostic A - Les défis de gestion de l'eau et des écosystèmes à relever en commun, CBLT, N’Djamena, Tchad, 177 pp., 2010.

Direction de la Statistique et de la Comptabilité Nationale (DSCN) : Conditions de vie des ménages et profil de pauvreté à l'Extrême-Nord Cameroun en 2001, Édit. Direction de la Statistique et de la Comptabilité Nationale, étude réalisée dans la cadre du PREPAFEN, 131 pp., accessible à l'adresse suivante : http://catalog.ihsn.org/catalog/3229/download/47794/ ECAM2-ProfilExtrOme-Nord.pdf (la date du dernier accès : 25 janvier 2020), 2002.

Institut National de la Statistique du Cameroun (INS) : Rapport national sur les objectifs du millénaire pour le développement en 2015, Institut National de la Statistique, Yaoundé, Cameroun, 48 pp., 2015.

Lang, C. et Gille, E. : Une méthode d'analyse du tarissement des cours d'eau pour la prévision des débits d'étiage, Norois, 201, 31-43, https://doi.org/10.4000/norois.1743, 2006.

Lévêque, C. : Variabilité du climat et des régimes hydrologiques, in : Les poissons des eaux continentales africaines. Diversité, écologie, utilisation par l'homme, édité par : Lévêque, C. et Paugy, D., IRD, Paris, accessible à l'adresse suivante : https://horizon.documentation.ird.fr/exl-doc/pleins_ textes/ed-06-08/010037336.pdf (la date du dernier accès : 14 mars 2019), 2006.

L'Hôte, Y. : Climatologie, in : Atlas de la province de L'ExtrêmeNord du Cameroun, IRD, Paris, 17-20, accessible à l'adresse suivante : https://books.openedition.org/irdeditions/11550?lang=fr (la date du dernier accès : 25 novembre 2020), 2000.

Loup, J. : Pardé Maurice. - Sur la puissance des crues en diverses parties du monde, Rev. Géogr. Alp., 53, 157-159, 1965.

Nguimalet, C. R. : Changements enregistrés sur les extrêmes hydrologiques de l'Oubangui à Bangui (République centrafricaine) : 
analyse des tendances, Revue des Sciences de 1'Eau, 30, 183196, 2017.

Olivry, J. C. : Fleuves et Rivières du Cameroun, collection "Monographies hydrologiques ORSTOM", vol. 9, MESRES/ORSTOM, Paris, 733 p. + cartes hors texte, accessible à l'adresse suivante : https://horizon.documentation.ird.fr/exl-doc/pleins_ textes/pleins_textes_6/Mon_hydr/25393.pdf (la date du dernier accès : 14 mars 2019), 1986.

Olivry, J. C. : Long term effects of rain shortage : the ill rivers of Western and Central Africa, in : FRIEND General Report/UNESCO IHP V, UNESCO, Paris, 158-162, 1997.

Olivry, J. C., Bricquet, J. P., Bamba, F., et Diarra, M. : Le régime hydrologique du Niger supérieur et le déficit des deux dernières décennies, in : Quelques données préliminaires sur l'environnement et la qualité des apports du Niger au Sahel, édité par : OLIVRY, J. C., Diallo Iam, M., et Bricquet, J. P., ORSTON-CNRST, Bamako, 9-25, 1994.
Olivry, J. C., Chouret, A., Vuillaume, G., Lemoalle, J., et Bricquet, J. P. : Hydrologie du lac Tchad, ORSTOM, Paris, 302, accessible à l'adresse suivante : https://core.ac.uk/download/pdf/39853684. pdf (la date du dernier accès : 6 novembre 2020), 1996.

Olivry, J. C., Bricquet, J. P., et Mahé, G. : Variabilité de la puissance des crues des grands cours d'eau d'Afrique intertropicale et incidence de la baisse des écoulements de base au cours des deux dernières décennies, in : Water resources variability in Africa during the XXth Century, actes de colloque (Abidjan, Côte d'Ivoire), édité par : Servat, É., Hughes, D., Fritsch, J. M., et Hulme, M., IAHS, 252, 189-197, 1998. 